\title{
Adhering to best practice guidelines in outcomes measurement
}

*David Morley \& Crispin Jenkinson

Nuffield Department of Population Health, University of Oxford, Old Road Campus, Oxford OX3 7LF, United Kingdom

*Corresponding author:

David Morley

Health Services Research Unit

Richard Doll Building

Nuffield Department of Population Health

University of Oxford

Old Road Campus

Oxford OX3 7LF

United Kingdom

Tel: +44 (0) 1865289432

Email: david.morley@dph.ox.ac.uk

Keywords: FDA guidelines; outcomes measurement; patient reported outcome measure; PDQ-39; PROM 
We read with great interest the recent review of Asakawa et al (2016). In their opening section the authors review what they term as 'subjective assessments for motor symptoms' in which they include a number of patient reported outcome measures (PROMs), one of which is the 39-item Parkinson's Disease Questionnaire (PDQ-39). As developers of the PDQ39 (Peto et al, 1995; Jenkinson et al, 2012) and its e-based equivalent the ePDQ (Morley et al, 2014; Morley et al 2015), our attention was initially drawn to some rather striking errors in one of the tables in the article. Under Table 1, the PDQ-39 is listed as a 'scale for subjective evaluation of motor symptoms'. The PDQ-39 is, in fact, a measure of health related quality of life and has been used as such in over 500 trials and studies listed in PubMed. Additionally, the PDQ-39 is described as 'somewhat complicated'. There is no evidence for this as both the PDQ-39 and ePDQ have been shown to be highly acceptable to respondents (Jenkinson et al, 2012; Morley et al, 2014). Most alarmingly, the authors state that a weakness of the PDQ-39 is that users 'should be careful to avoid observation bias'. This implies that the PDQ-39 is a clinician administered measure, whereas the PDQ-39 is actually a PROM, thereby designed for self-completion by people with Parkinson's.

On further reflection we believe there is a more fundamental issue that the review fails to address, and one that is likely to dominate both the future development and use of assessment tools; the importance of adherence to best practice guidelines for PROMs to be included in clinical trials. Stringent guidance for both developers and users of such PROMs are now in place. The most prominent of these are provided by the United States Food and Drug Administration (FDA: 2009), but similar guidelines have also been published by, for example, the European Medicines Agency (2004). Other guidance such as the COnsensusbased Standards for the selection of health Measurement INstruments (COSMIN) also exists, 
although their focus is not specifically on clinical trials. That said, COSMIN should be recognised as an important tool for assessing the quality of PROMs.

Such has been their influence, some have now suggested that FDA guidelines should be considered as best practice, regardless of whether a PROM is being incorporated into, or developed for, trials supporting pharmaceutical label claims (Speight \& Barendse 2010). Future reviews such as that of Asakawa et al (2016) might consider furnishing their readers as to whether the measures reviewed adhere to such guidelines.

In brief, the FDA expects the following of PROMs used in clinical trials:

- A well-documented record of development that is accessible via open-access peer reviewed publications.

- Evidence of strong content validity through the involvement of patients and relevant experts during the development process.

- Evidence of appropriate levels of internal and external reliability.

- Evidence of 'other validity' such as construct, concurrent, discriminant and knowngroups validity.

- The ability to detect meaningful change in respondents' health state.

Some established measures, such as the PDQ-39, broadly adhere to such guidance, although many do not. This is particularly the case with regard to patient involvement in the development of measures, and particularly so in item-generation. For example, the field of disability and rehabilitation measurement is notoriously poor when it comes to FDA 
compliant measures (Morley et al, 2014). Newer measures, however, such as the Oxford Participation and Activities Questionnaire (Ox-PAQ; Morley et al, 2016; Kelly et al, 2015; Morley et al, 2014) have been forensic in following FDA guidance from initial development through to the fully validated measure. Following such a pathway makes the Ox-PAQ far more likely to be included in important clinical trials.

This leads to a brief but important conclusion. When incorporating PROMs into clinical trials, users have to ask themselves one overriding and critical question; is this instrument FDA compliant? If the answer is no, we strongly recommend finding one that is. We also suggest that when conducting systematic reviews of established outcome measures, reviewers might consider using criteria such as those provided by the FDA as part of the process.

Funding: No funding supported this article.

Conflict of interest: $\mathrm{CJ}$ is a developer of the PDQ-39 and receives royalties from its use. Both $\mathrm{CJ}$ and DM have undertaken consultancy work for Oxford University Innovation who hold the licence for the PDQ-39.

\section{References}

Asakawa, T., Fang, H., Sugiyama, K., Nozaki, T., Kobayashi, S., Hong, Z., Suzuki, K., Mori, N., Yang, Y., Hua, F., Ding, G., Wen, G., Namba, H., Xia, Y., 2016. Human behavioral assessments in current research of Parkinson's disease. Neurosci. Biobehav. Rev. 68, $741-772$. 
COnsensus-based Standards for the selection of health Measurement INstruments (COSMIN)

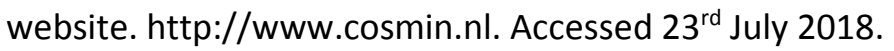

EMA., 2004. Reflection paper on the regulatory guidance for the use of health-related quality of life (HRQL) measures in the evaluation of medicinal products. EMEA/CHMP/EWP139391/2004.

Food and Drug Administration, Department of Health and Human Sciences., 2009. Guidance to Industry. Patient Reported Outcome Measures. Use in Medical Product Development to Support Labelling Claims. Silver Spring, MD: Food and Drug Administration.

Jenkinson, C., Fitzpatrick, R., Peto, V., Dummett, S., Morley, D., Saunders, P., 2012. The Parkinson's Disease Questionnaire: User Manual, 3rd Edition. Oxford: Isis Outcomes.

Kelly, L., Jenkinson, C., Dummett, S., Dawson, J., Fitzpatrick, R., Morley, D., 2015. Development of the Oxford Participation and Activities Questionnaire: Constructing an item pool. Patient. Relat. Outcome. Meas. 6, 145-155.

Morley, D., Dummett, S., Kelly, L., Dawson, J., Fitzpatrick, R., Jenkinson, C., 2016. Validation of the Oxford Participation and Activities Questionnaire. Patient. Relat. Outcome. Meas. 7, 73-80.

Morley, D., Dummett, S., Kelly, L., Dawson, J., Jenkinson, C., 2015. Evaluating the psychometric properties of an e-based version of the 39-item Parkinson's Disease Questionnaire. Health. Qual. Life. Outcomes. 13:5.

Morley, D., Dummett, S., Kelly, L., Dawson, J., Fitzpatrick, R., Jenkinson, C., 2014. The Oxford Participation and Activities Questionnaire: Study Protocol. Patient. Relat. Outcome. Meas. 5, 1-6. 
Morley, D., Dummett, S., Kelly, L., Dawson, J., Jenkinson, C., 2014. An electronic version of the PDQ39: acceptability to respondents and assessment of alternative response formats. J. Parkinsons. Dis. 4, 467-472.

Speight, J., Barendse, S.M., 2010. FDA guidance on patient reported outcomes: a prompt for the industry to raise scientific standards. BMJ. 340:c2921. 